\title{
The benefit of using bladder sub-volume equivalent uniform dose constraints in prostate intensity-modulated radiotherapy planning
}

This article was published in the following Dove Press journal:

OncoTargets and Therapy

12 December 2016

Number of times this article has been viewed

\author{
Jian Zhu ${ }^{1-3}$ \\ Antoine Simon ${ }^{3-5}$ \\ Pascal Haigron ${ }^{3-5}$ \\ Caroline Lafond ${ }^{4-6}$ \\ Oscar Acosta ${ }^{4,5}$ \\ Huazhong Shu ${ }^{1,3}$ \\ Joel Castelli ${ }^{4-6}$ \\ Baosheng $\mathrm{Li}^{1-3}$ \\ Renaud De Crevoisier ${ }^{3-6}$ \\ 'Laboratory of Image Science and \\ Technology, Southeast University, \\ Nanjing, Jiangsu, ${ }^{2}$ Department of \\ Radiation Oncology, Shandong \\ Cancer Hospital \& Institute, \\ Jinan, ${ }^{3}$ Centre de Recherche en \\ Information Biomédicale Sino- \\ français, Nanjing, People's Republic \\ of China; ${ }^{4}$ Institut National de la \\ Sante et de la Recherche Medicale, \\ U 1099, ${ }^{5}$ Laboratory of Signal and \\ Image Processing (LTSI), University \\ of Rennes I, ' Department of \\ Radiotherapy, Centre Eugène \\ Marquis, Rennes, France
}

Background: To assess the benefits of bladder wall sub-volume equivalent uniform dose (EUD) constraints in prostate cancer intensity-modulated radiotherapy (IMRT) planning.

Methods: Two IMRT plans, with and without EUD constraints on the bladder wall, were generated using beams that deliver 80 Gy to the prostate and 46 Gy to the seminal vesicles and were compared in 53 prostate cancer patients. The bladder wall was defined as the volume between the external manually delineated wall and a contraction of $7 \mathrm{~mm}$ apart from it. The bladder wall was then separated into two parts: the internal-bladder wall (bla-in) represented by the portion of the bladder wall that intersected with the planning target volume (PTV) plus $5 \mathrm{~mm}$ extension; the external-bladder wall (bla-ex) represented by the remaining part of the bladder wall. In the IMRT plan with EUD constraints, the values of " $a$ " parameter for the EUD models were 10.0 for bla-in and 2.3 for bla-ex. The plans with and without EUD constraints were compared in terms of dose-volume histograms, 5-year bladder and rectum normal tissue complication probability values, as well as tumor control probability (TCP) values.

Results: The use of bladder sub-volume EUD constraints decreased both the doses to the bladder wall ( $V_{70}: 22.76 \%$ vs $19.65 \%, D_{\text {mean }}: 39.82 \mathrm{~Gy}$ vs $\left.35.45 \mathrm{~Gy}\right)$ and the 5-year bladder complication probabilities ( $\geq$ LENT/SOMA Grade 2: $20.35 \%$ vs $17.96 \%$; bladder bleeding: $10.63 \%$ vs $8.64 \%)$. The doses to the rectum wall and the rectum complication probabilities were also slightly decreased by the EUD constraints compared to physical constraints only. The minimal dose and the $V_{76 \mathrm{~Gy}}$ of $\mathrm{PTV} \mathrm{V}_{\text {prostate }}$ were, however, slightly decreased by EUD optimization, nevertheless without significant difference in TCP values between the two plans, and the PTV parameters finally respected the Groupe d'Etude des Tumeurs Uro-Génitales recommendations.

Conclusion: Separating the bladder wall into two parts with appropriate EUD optimization may reduce bladder toxicity in prostate IMRT. Combining biological constraints with physical constraints in the organs at risk at the inverse planning step of IMRT may improve the dose distribution.

Keywords: prostate, IMRT, equivalent uniform dose

\section{Introduction}

The EUD was proposed by Niemierko in 1997 to convert a nonuniform partial dose distribution into an EUD distribution with the same cancer killing effect. In 1999, it was extended to gEUD to describe doses on normal tissues which is more comprehensive than a single dose-volume threshold for a unified description compared to the nonuniform dose distribution. ${ }^{1}$ Furthermore, since EUD can be used to describe the biological dose-volume characteristics of normal tissues by setting different parameters, it may also be used as biological constraint for IMRT optimization to
Correspondence: Jian Zhu

Laboratory of Image Science and Technology, Southeast University, Sipailou 2\#, Nanjing, Jiangsu Province, 210096, People's Republic of China

$\mathrm{Tel} / \mathrm{fax}+8653167627907$

Email zhujian.cn@।63.com 
spare normal tissue complications. ${ }^{2,3}$ Indeed, Wu et $\mathrm{al}^{4}$ argued that the use of merely physical objective functions (ie, dose-volume constraints) in IMRT optimization may not be fully adequate to reduce toxicity, since it does not reflect the nonlinear radio-induced normal tissue reaction, especially when the dose distribution is not uniform. Optimizing the IMRT objective function with EUD biological constraints, ie, limiting particularly the dose levels highly correlated with toxicity, may therefore strongly help to reduce the toxicity. The application of EUD constraints implies, however, the choice of a specific " $a$ " parameter for each kind of toxicity or organ. This parameter " $a$ ", the only one within the EUD model, is related to the " $n$ " parameter of the LKB NTCP model with the relationship $a=1 / n .{ }^{5}$ For serial organs, such as the spinal cord or the rectum, $a>1$ means that toxicity is mainly linked to high doses (hot spots), which are therefore to be considered with high priority at the inverse planning step of IMRT. For parallel organs, such as parotid, lung, liver and kidney, " $a$ " close to zero means that the mean dose and cold and hot spots can be equally considered for inverse planning. ${ }^{1,6}$

With regard to prostate radiotherapy, the two most frequent toxicities are linked to the rectum and bladder irradiation. Indeed, due to the proximity of the anatomical structures, the posterior and the superior part of the PTV (with margins $\geq 5 \mathrm{~mm}$ ) overlapped with the rectum and the bladder wall, respectively, generating a conflict to respect the dose constraints in both the PTV and the OARs. The amount of overlap volume between PTV and bladder or rectum adversely affects sparing of that organ, though other metrics of plan quality are less affected. ${ }^{7}$ A medical choice needs to balance either in favor of limiting toxicity or toward increasing local control. Depending on the prescribed total dose to the prostate, which can range from 74 to $80 \mathrm{~Gy}$, and in order to spare more OARs, some compromise with a lower dose to the PTV overlapped with rectum and bladder, ${ }^{8}$ while some may also assign different physical dose constraints to the OARs by subdividing the target area into "inside" and "outside" regions. ${ }^{9}$ However, specific biological constraints could be used in these PTV-OAR overlapped sub-volumes to optimize this compromise.

Although the " $n$ " value of the EUD model is relatively well identified for the rectal toxicity (range: $0.06-0.24$ ), ${ }^{10}$ the " $n$ " value for the bladder is still intangible due to its geometric uncertainties. ${ }^{11}$ The majority of the studies ${ }^{12-20}$ suggest bladder as a "serial" organ, its toxicity, mainly urgency and obstruction, being related to the highest dose delivered within the trigone region. On the other hand, some studies $^{21-23}$ found bladder as a mixed "serial-parallel" organ because severe urinary toxicity is related to both low (10 Gy) and high (80 Gy) doses delivered to the bladder. The QUANTEC (QUAntitative Normal TissuE models in the Clinic) $)^{24}$ investigation concluded "prudently" that both maximum dose and a relatively large irradiated bladder volume (50\%) may correlate with bladder toxicity (Grade $\geq 3$ late RTOG [Radiation Therapy Oncology Group]).

In this study, we assumed that bladder toxicities may be linked with differently irradiated bladder sub-volumes. The goal of this dosimetry study was to quantify the benefit of using such multiple bladder sub-volume EUD constraints at the inverse planning step of IMRT to decrease bladder toxicity in prostate cancer IMRT.

\section{Materials and methods}

A total of 53 patients having received IMRT for intermediateand high-risk localized prostate adenocarcinoma were included in this dosimetry study. Ethical approval was not required by the institutional review board (IRB) of Centre Eugène Marquis, because all the patients enrolled in this study had finished radiotherapy. Only the CT images of these patients were used as the materials in this experiment. All the patients have given their consents on the usage of their CT images in this study.

\section{CT acquisition and region of interest delineation}

All patients were immobilized in the supine position with an individualized vacuum bag during the $\mathrm{CT}$ simulation and treatment. A thin rectal catheter was used to improve the visibility of the anal canal and the rectum. An intravenous iodine contrast $\mathrm{CT}$ scan was acquired from the sacroiliac joints to the lower edge of the small trochanters with a slice thickness of 3-5 mm.

The OARs were defined according to the GETUG recommendations. ${ }^{25}$ The external contour of bladder was manually delineated. For the rectum, the external contour was delineated from $2 \mathrm{~cm}$ above to $2 \mathrm{~cm}$ below the CTV (clinical target volume). The bladder and rectum walls were obtained by regular two-dimensional contractions of 7 and $5 \mathrm{~mm}$, respectively.

The bladder wall was then separated into two parts as shown in Figure 1: bla-in represents the part of the bladder wall overlapped with the area corresponding to the $\mathrm{PTV}_{\text {prostatesv }}$ plus a $5 \mathrm{~mm}$ extension and bla-ex represents the remaining part of the bladder wall (outside the area

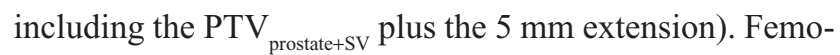
ral heads were finally delineated up to the upper edge of the low trochanters. 


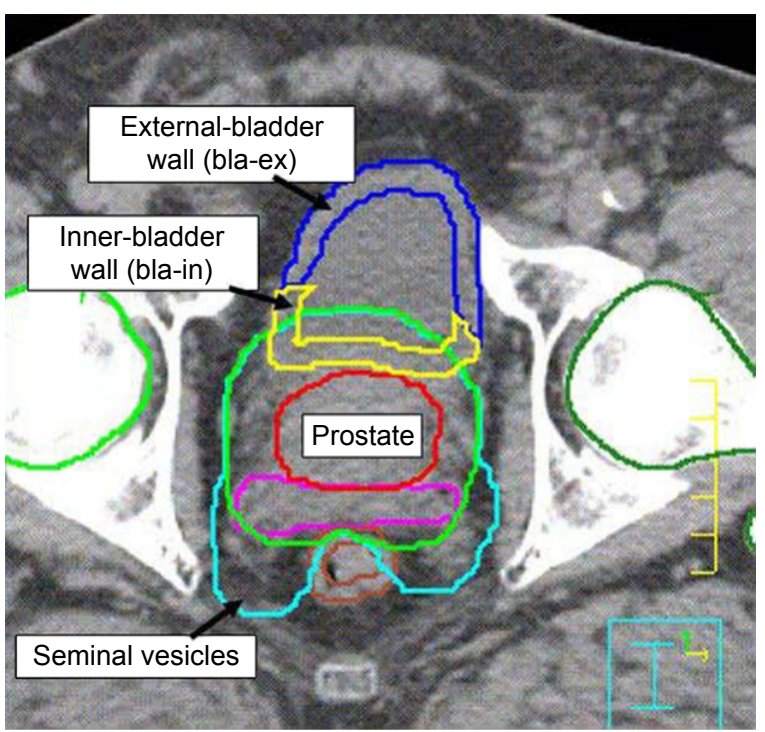

Figure I Delineation of the organs of interest.

Notes: The bladder wall was divided into two parts: the internal-bladder wall (bla-in, yellow lines) and the external-bladder wall (bla-ex, blue lines). The red line corresponds to the prostate and the green one to the prostate PTV. The purple line corresponds to the seminal vesicles (SV) and the cyan one to the SV PTV. Abbreviation: PTV, planning target volume.

\section{EUD parameters and IMRT planning}

The IMRT plans were generated by using ten $18 \mathrm{MV}$ beams delivering 80 Gy to the prostate and 46 Gy to the SV in two sequential phases. Phase 1 delivered 46 Gy $(2.0 \mathrm{~Gy} / \mathrm{fr}$ in 23 fr) with 5 coplanar beams $\left(36^{\circ}, 100^{\circ}, 180^{\circ}, 260^{\circ}, 324^{\circ}\right)$

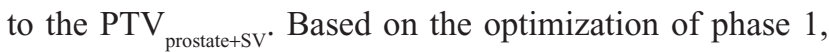
phase 2 used another five beams that delivered a boost of

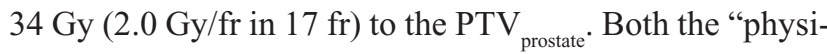
cal" IMRT plan (without EUD constraints; "Plan ${ }_{\text {phys }}$ ") and the "biological" IMRT plan (with EUD constraints, "Plan bio ") were generated for each of the 53 patients, with Philips Pinnacle 3 v9.2 TPS.

For Plan $_{\text {phys }}$, the dose constraints were defined according to the "GETUG" ${ }^{26}$ recommendations: minimum dose $>72$ Gy and $V_{76 \mathrm{~Gy}}>95 \%$ for the $\mathrm{PTV}_{\text {prostate }}$; maximum dose (within $1.8 \mathrm{~mL})<80$ Gy and $V_{70 \mathrm{~Gy}} \leq 50 \%$ for the bladder wall; maximum dose (within $1.8 \mathrm{cc}$ ) $<76$ Gy and $V_{72 \mathrm{~Gy}} \leq 25 \%$ for the rectal wall; and $V_{55 \mathrm{~Gy}}<5 \%$ for the femoral heads.

For Plan ${ }_{\text {bio }}$, dose constraints were derived from the corre-

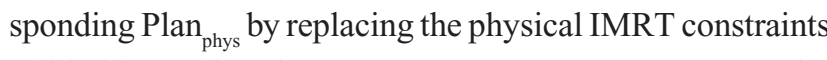
of bladder wall with two "Max EUD" constraints, namely, $\mathrm{EUD}_{\text {bla-in }}$ and $\mathrm{EUD}_{\text {bla-ex }}$, respectively, in a sequential approach. To evaluate the effect of EUD, only penalty weight and the objective EUD values were adjusted during the IMRT optimization process of Plan bio The toxicity of the "bla-in" was considered to be correlated with high dose delivered in a small volume, whereas for the "bla-ex", a large irradiated volume was considered as being correlated with toxicity.
Thus, the corresponding EUD bla-in $_{\text {and }}$ EUD ${ }_{\text {bla-ex }}$ parameters were set to " $a=10.0 " 19$ and " $a=2.3$ ", respectively. ${ }^{27,28}$ During the IMRT optimization process of Plan $_{\text {bio }}$, if the dose to the rectal wall was significantly increased, another "Max EUD" constraint was added for the rectal wall, with the parameter " $a=5.0$ ". ${ }^{29}$

\section{Dosimetry end points and statistical analysis}

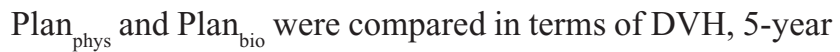
bladder and rectal NTCP values (overall toxicity and bleeding episode) and TCP values. To visually present the effect on dose-volume variation, the integral DVH curves of two targets $\left(\mathrm{PTV}_{\text {prostate }}, \mathrm{PTV}_{\text {prostate+SV}}\right.$ ) and four OARs (rectal wall, bladder wall, bla-ex and bla-in) were averaged from both Plan phys $_{\text {and }}$ Plan $_{\text {bio }}$ of all the 53 patients. These average DVH curves were calculated based on the DVH files exported from Pinnacle TPS $^{30}$ and were plotted using "Matlab" software (The MathWorks, Inc., Natick, MA, USA version 8.0). The DVH end points included $D_{2 \%}, D_{50 \%}$, $D_{95 \%}, D_{98 \%}$ and $D_{\text {mean }}$ of $\mathrm{PTV}_{\text {prostate }}, V_{70 \mathrm{~Gy}}, D_{\text {max }}$ and $D_{\text {mean }}$ of bladder, and $V_{72 \mathrm{~Gy}}, D_{\max }$ and EUD of rectum, according to the ICRU Report $83 .{ }^{31}$ The NTCP values for bladder and rectal 5-year toxicities were calculated using an LKB model with the following parameters: $\operatorname{TD}_{50}(1)=78.68 \mathrm{~Gy}, \mathrm{n}=0.09$, $m=0.17$ for bladder $\geq$ Grade 2 LENT/SOMA toxicity and $\mathrm{TD}_{50}(1)=85.31 \mathrm{~Gy}, \mathrm{n}=0.36, m=0.30$ for bladder bleeding; ${ }^{19}$ $\mathrm{TD}_{50}(1)=80.46 \mathrm{~Gy}, \mathrm{n}=0.01, m=0.12$ for rectal $\geq$ Grade 2 LENT/SOMA toxicity and $\mathrm{TD}_{50}(1)=82.14 \mathrm{~Gy}, \mathrm{n}=0.02$, $m=0.12$ for rectal bleeding. ${ }^{32}$

To estimate the relative difference in tumor control which might reasonably be predicted between two not too dissimilar situations, the TCP values were calculated according to the model proposed by Fowler ${ }^{33}$ as follows:

$$
T C P_{L Q}(D)=\exp \left[-N_{c l o} \sum_{k=1}^{N} \mathrm{v}_{k} S F_{L Q}\left(D_{k}\right)\right]
$$

with

$$
S F_{L Q}\left(D_{k}\right)=\exp \left(-\alpha D_{k}-\beta D_{k}^{2} / n\right)
$$

where $N_{c l o}$ is the total initial number of clonogenic cells, $S F_{L Q}\left(D_{k}\right)$ is the surviving fraction of cells exposed to dose $D_{k}$ in voxel $k, v_{k}$ is the relative volume of voxel $k, \alpha$ is the intrinsic radiosensitivity representing the nonrepairable radiation damage, $\beta$ represents a repairable type of injury that is responsible for the dose-per-fraction effect and $n$ is the number of treatment fractions. The following parameters were chosen: $N_{c l o}=138,{ }^{34} \alpha=0.0391 \mathrm{~Gy}^{-1}$ and $\beta=0.0263 \mathrm{~Gy}^{-2}{ }^{35}$ 
In order to quantitatively assess the target coverage, the $\mathrm{CI}$ and $\mathrm{HI}$ were calculated for $\mathrm{PTV}_{\text {prostate }}$. The $\mathrm{CI}$ and $\mathrm{HI}$ were defined as follows:

$$
\mathrm{CI}=\frac{V_{t, r e f}}{V_{t}} \cdot \frac{V_{t, r e f}}{V_{r e f}}
$$

where $V_{t}$ is the volume of tumor, $V_{t, r e f}$ is the volume of tumor surrounded by the reference dose curve and $V_{\text {ref }}$ is the volume of the reference dose curve. CI therefore varies between 0 and 1 . A larger CI value indicates a better conformal dose distribution; ${ }^{36}$

$$
\mathrm{HI}=\frac{D_{2}-D_{98}}{D_{p}}
$$

where $D_{2}$ stands for the dose on $2 \%$ volume of the tumor and $D_{98}$ stands for the dose on $98 \%$ volume of the tumor. Therefore, $D_{2}$ and $D_{98}$ describe the maximum and minimum dose on the tumor target, respectively. $D_{p}$ is the prescription dose. $\mathrm{HI}$ varies between 0 and 1 . The smaller HI value indicates a more homogeneous dose distribution on the tumor target. ${ }^{37}$

To determine the statistical significance, the Mann-Whitney test was performed and $P$-value $<0.05$ was considered to be statistically significant. All calculations were performed using the SPSS program, version 16.0.2 (SPSS Inc., Chicago, IL, USA).

\section{Results}

The integral average DVH values of tumor target and normal tissues for the 53 patients are plotted in Figure 2, where $x$ - and $y$-axes indicate absolute dose (in "Gy") and normalized percentage volume (\%), respectively, with the solid curves corresponding to Plan ${ }_{\text {phys }}$ and the dashed curves corresponding to Plan $_{\text {bio }}$. The statistical comparison of the two plans is shown in Table 1.

Both Table 1 and Figure 2 show that compared to Plan ${ }_{\text {phys }}$, Plan $_{\text {bio }}$ decreased significantly the bladder doses, in terms of bla-in, bla-ex and the whole bladder wall, as well as the complication probabilities. With regard to the rectal wall,

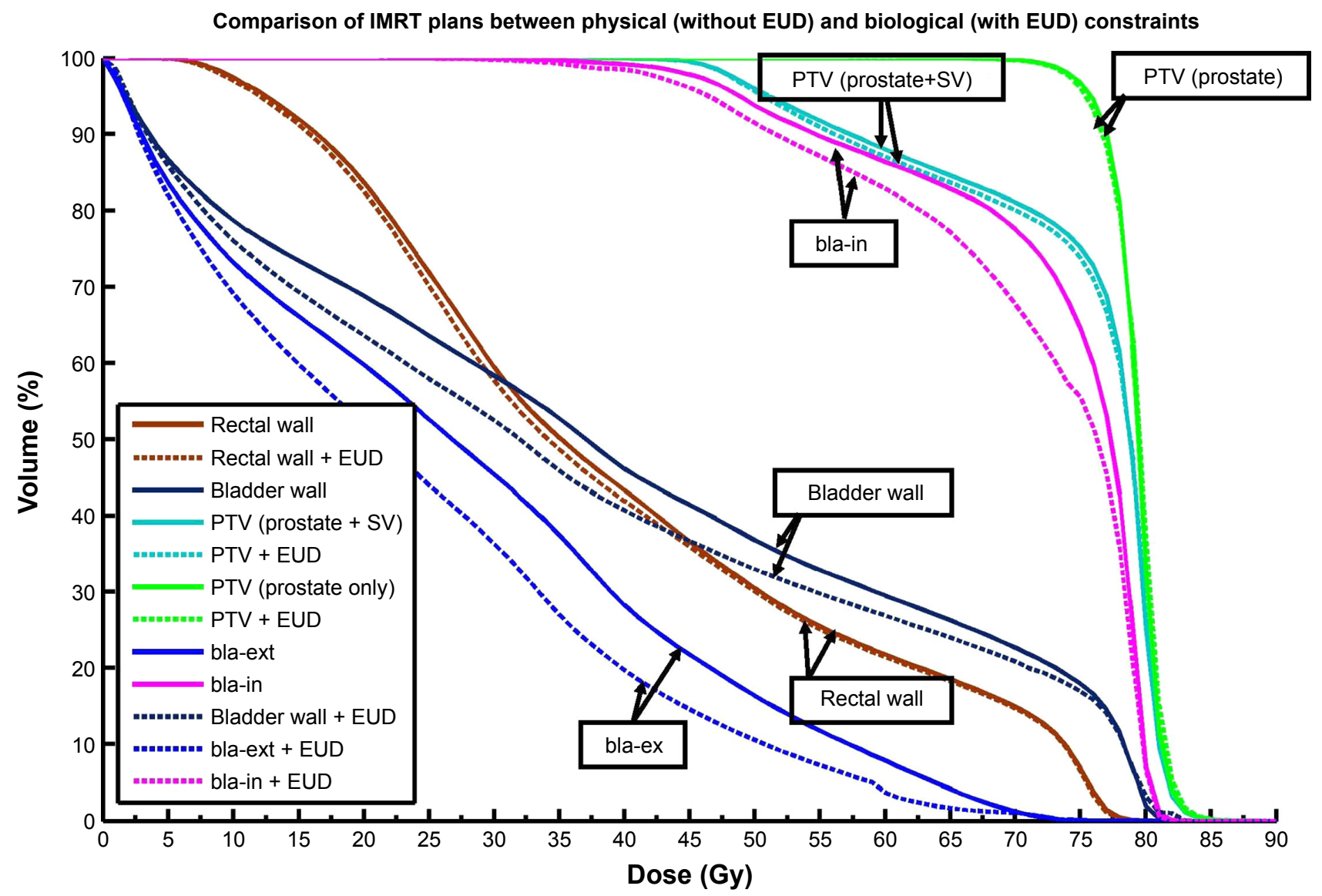

Figure 2 Mean DVH of the physical plan and biological EUD plan.

Abbreviations: IMRT, intensity-modulated radiotherapy; bla-in, internal-bladder wall; bla-ex, external-bladder wall; DVH, dose-volume histograms; SV, seminal vesicles; EUD, equivalent uniform dose; PTV, planning target volume. 
Table I Statistical comparison between plans without and with EUD IMRT

\begin{tabular}{|c|c|c|c|c|}
\hline $\begin{array}{l}\text { Organs } \\
\text { at risk }\end{array}$ & Factors & $\begin{array}{l}\text { Plan without EUD } \\
\text { optimization (Plan }_{\text {phys }} \text { ) }\end{array}$ & $\begin{array}{l}\text { Plan with EUD } \\
\text { optimization }\left(\operatorname{Plan}_{\text {bio }}\right)\end{array}$ & $P$-value \\
\hline \multirow[t]{10}{*}{ PTV } & $D_{\min }{ }^{c}$ & $92.99 \% \pm 3.35 \%$ & $92.70 \% \pm 3.50 \%$ & 0.008 \\
\hline & $V_{76 \mathrm{~Gy}^{a}}$ & $96.10 \% \pm 2.15 \%$ & $95.15 \% \pm 2.46 \%$ & $<0.001$ \\
\hline & $D_{2 \%}{ }^{b}$ & $83.60 \pm 2.91 \mathrm{~Gy}$ & $83.79 \pm 2.86 \mathrm{~Gy}$ & 0.125 \\
\hline & $D_{50 \%}$ & $80.38 \pm 1.93$ Gy & $80.43 \pm 1.91$ Gy & 0.554 \\
\hline & $D_{95 \%}$ & $76.75 \pm 1.75 \mathrm{~Gy}$ & $76.85 \pm 2.20 \mathrm{~Gy}$ & 0.297 \\
\hline & $D_{98 \%}$ & $74.92 \pm 1.81 \mathrm{~Gy}$ & $75.06 \pm 2.32 \mathrm{~Gy}$ & 0.330 \\
\hline & $D_{\text {mean }}$ & $79.50 \pm 1.64 \mathrm{~Gy}$ & $79.42 \pm \mathrm{I} .4 \mathrm{I} \mathrm{Gy}$ & 0.554 \\
\hline & $\mathrm{Cl}$ & $0.74 \pm 0.04$ & $0.94 \pm 0.02$ & $<0.001$ \\
\hline & $\mathrm{HI}$ & $0.10 \pm 0.02$ & $0.11 \pm 0.02$ & $<0.00$ I \\
\hline & $\mathrm{TCP}$ & $91.12 \% \pm 0.48 \%$ & $91.10 \% \pm 0.41 \%$ & 0.972 \\
\hline \multirow[t]{5}{*}{ Rectal wall } & $D_{\max }{ }^{d}$ & $75.90 \pm 2.04 \mathrm{~Gy}$ & $75.19 \pm 2.17 \mathrm{~Gy}$ & 0.003 \\
\hline & $V_{72 G y}$ & $14.35 \% \pm 5.26 \%$ & $14.02 \% \pm 5.04 \%$ & 0.067 \\
\hline & EUD $(a=5.0)$ & $55.94 \pm 3.6 \mathrm{I} \mathrm{Gy}$ & $55.6 \mathrm{I} \pm 3.44 \mathrm{~Gy}$ & 0.001 \\
\hline & NTCP $5 y R T^{e}$ & $22.77 \% \pm 13.04 \%$ & $19.93 \% \pm 7.09 \%$ & 0.199 \\
\hline & NTCP $5 y R b f^{f}$ & $29.51 \% \pm 9.24 \%$ & $27.44 \% \pm 8.10 \%$ & 0.217 \\
\hline \multirow[t]{5}{*}{ Bladder wall } & $D_{\max }{ }^{d}$ & $79.96 \pm 0.84 \mathrm{~Gy}$ & $79.63 \pm 0.81 \mathrm{~Gy}$ & $<0.00$ I \\
\hline & $V_{70 G y}$ & $22.76 \% \pm 10.59 \%$ & $19.65 \% \pm 9.96 \%$ & $<0.00 \mathrm{I}$ \\
\hline & $D_{\text {mean }}$ & $39.82 \pm 12.73 \mathrm{~Gy}$ & $35.45 \pm 12.58 \mathrm{~Gy}$ & $<0.001$ \\
\hline & NTCP 5уBTg & $20.35 \% \pm 6.70 \%$ & $17.96 \% \pm 6.36 \%$ & $<0.00 \mathrm{I}$ \\
\hline & NTCP $\left.5 y B b\right|^{h}$ & $10.63 \% \pm 6.29 \%$ & $8.64 \% \pm 5.50 \%$ & $<0.00 \mathrm{I}$ \\
\hline bla-exi & EUD $(a=2)$ & $33.48 \pm 8.93 \mathrm{~Gy}$ & $27.50 \pm 8.68 \mathrm{~Gy}$ & $<0.00 \mathrm{I}$ \\
\hline bla-ini & EUD $(a=10)$ & $73.30 \pm 3.37 \mathrm{~Gy}$ & $71.23 \pm 3.71 \mathrm{~Gy}$ & $<0.001$ \\
\hline $\mathrm{FH}^{\mathrm{k}}$ & $V_{55 G y}$ & $0.04 \% \pm 0.18 \%$ & $0.20 \% \pm 0.59 \%$ & 0.026 \\
\hline
\end{tabular}

Notes: ${ }^{a} V_{76 G y}$ corresponds to the $95 \%$ prescription dose on $\mathrm{PTV}_{\text {prostate }}{ }^{\mathrm{b}} \mathrm{D}_{2 \%}, \mathrm{D}_{50 \%}, \mathrm{D}_{95 \%}$ and $\mathrm{D}_{98 \%}$ are the minimum absorbed dose that covered $2 \%, 50 \%, 95 \%$ and $98 \%$ of the volume of PTV ${ }_{\text {prostate }}$, respectively; ' $D_{\text {min }}$ stands for the minimum dose on target which is the relative dose of target volume minus $1.8 \mathrm{cc}$; ${ }^{\mathrm{d}} D_{\text {max }}$ is the maximum dose on 1.8 cc volume; ${ }^{\text {55RT }}$ means the overall rectal toxicity $\geq$ LENT/SOMA Grade 2 at 5-year follow-up; ${ }^{31}$ f5yRb means the rectal bleeding event (one episode) at 5-year follow-up; ${ }^{31}$ ${ }^{8} 5$ yBT means the overall bladder toxicity $\geq$ LENT/SOMA Grade 2 at 5 -year follow-up; ${ }^{19}$ h5yBbl means the bladder bleeding event (one episode) at 5 -year follow-up; ${ }^{19}$ 'the external-bladder wall; 'the internal-bladder wall; kbilateral femoral head.

Abbreviations: $\mathrm{Cl}$, conformal index; IMRT, intensity-modulated radiotherapy; EUD, equivalent uniform dose; HI, homogeneity index; NTCP, normal tissue complication probability; PTV, planning target volume; TCP, tumor control probability; bla-in, internal-bladder wall; bla-ex, external-bladder wall; FH, femoral head; LENT/SOMA, late effects of normal tissues/subjective objective management analytic.

only $D_{\max }$ and EUD showed statistical significance while the dashed DVH line $\left(\right.$ Plan $\left._{\text {bio }}\right)$ is closely covered above the solid line $\left(\right.$ Plan $\left._{\text {phys }}\right)$ in the whole dose region, which at least indicates that the bladder sparing did not sacrifice rectal wall.

Plan $_{\text {bio }}$ also slightly decreased the PTV dose coverage considering $V_{76 \mathrm{~Gy}}$ and $D_{\text {min }}$ of $\mathrm{PTV} \mathrm{V}_{\text {prostate }}$, but still met the GETUG recommendations (more than 95\%). No significant difference was found in the TCP value.

Figure 3 visually illustrates the effect of EUD optimization on irradiation isodose distribution around prostate target and OARs in one of the 53 cases. It is obvious that, by using EUD constraint on bla-in, the high-dose line ( $110 \%$ of prescription dose, red line) was specifically "expelled" out of the bladder wall and fell on the prostate tumor target area. In addition, the high-dose line did not impair the internal side of rectal wall, and the low-dose line around bla-ex also contracted toward the tumor target. However, the corresponding effect is that the dosimetry homogeneity in the target decreased as price of sparing surrounding normal tissues

\section{Discussion}

As a "hollow" organ, the contents of the bladder may be irrelevant to estimate the risk of complications. Indeed, Harsolia et $\mathrm{al}^{21}$ argued that an analysis of the initial bladder wall volume, which is the actual biologic structure at risk, correlates more accurately with chronic genitourinary toxicity than the absolute solid volume of the bladder. In our study, only the dose distributed in the three-dimensional volume enclosed between the inner and outer surfaces of the bladder wall was optimized. This volume, belonging to the trigone area, appears to be less variable between and during the treatment fractions than the superior part of the bladder or the dome whose volume depends on the bladder filling.

The main innovation of this study is applying two EUD constraints with different parameters to separated bladder 


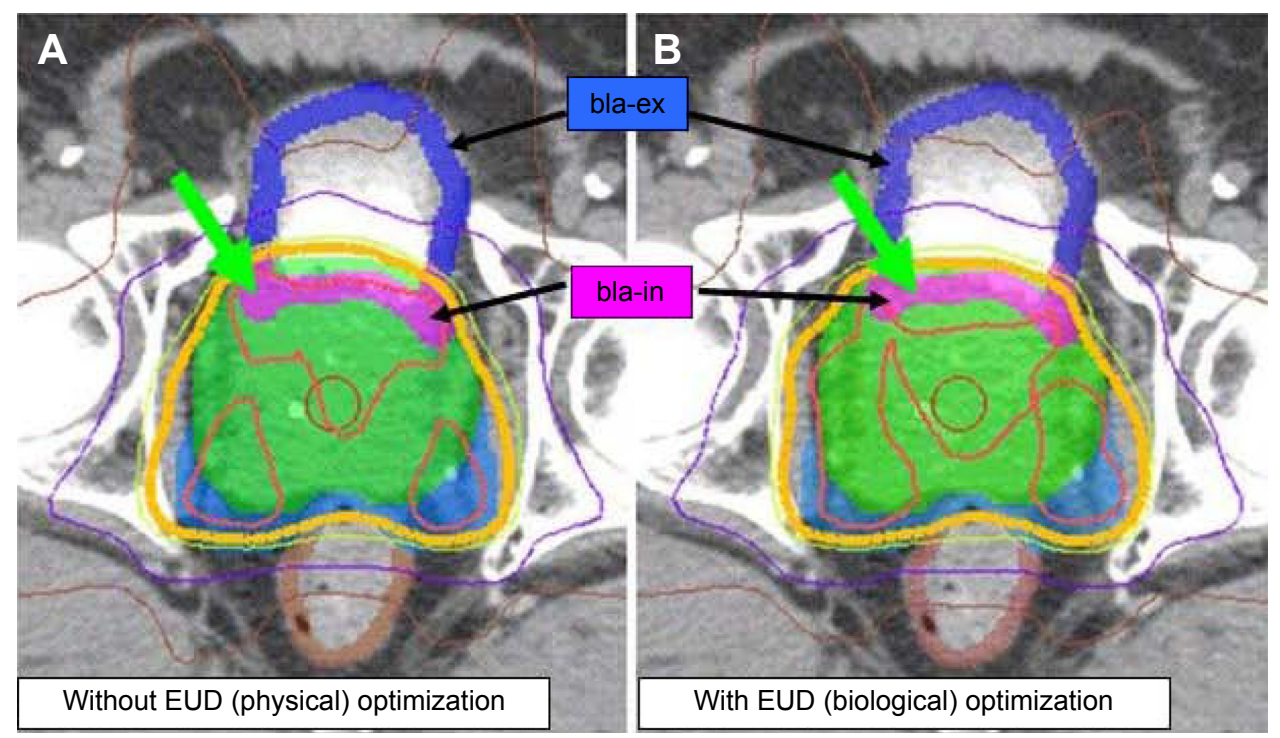

Figure 3 Dose distribution comparison on tumor target between plans with or without EUD IMRT optimization.

Notes: The left figure (A) presents the dose distribution without EUD IMRT optimization, while the right figure (B) presents the dose distribution with EUD IMRT optimization. The yellow dose curve covering prostate and seminal vesicle assign for the prescription dose 76 Gy of phase $\mathrm{I}$. The green arrows indicate that the higher dose (red curve, $80 \mathrm{~Gy}$ ) in the internal-bladder wall is expelled out of the bladder wall area. The green area corresponds to the prostate PTV.

Abbreviations: bla-in, internal-bladder wall; bla-ex, external-bladder wall; EUD, equivalent uniform dose; IMRT, intensity-modulated radiotherapy; PTV, planning target volume.

sub-volumes, which comes from the assumption that bladder toxicities may be linked with differently irradiated bladder sub-volumes. Most studies ${ }^{12-18}$ found that at least one of the primary mechanisms of urinary function impairment depends on the irradiation of a "small" volume (ie, the caudal portion of bladder unavoidably included in the PTV) to a "high" dose, which indicated a serial-like behavior. Marks et $\mathrm{al}^{38}$ found that the majority of the bladder can be irradiated with $\sim 30-50$ Gy without serious complications in contrast to a small fraction of the bladder that received $60-65$ Gy with serious complications. In our previous study, ${ }^{19}$ the estimated volume factor " $n$ " of LKB NTCP model for bladder was about 0.1 , which also pointed to the serial-like behavior. On the other hand, Harsolia et $\mathrm{al}^{21}$ found that a large number of points in bladder wall DVH $\left(V_{10-82}\right)$ correlated with chronic urinary toxicity and chronic urinary retention, which suggested a serial-parallel-like behavior. Thus, the bladder tissue located in low-dose area should also be considered by physicians and physicists. From the aforementioned debates, a single EUD parameter value to bladder may not be appropriate to present its serial-parallel characteristics in biological IMRT. Therefore, we assume the reason that although most commercial TPSs provide the biological IMRT optimization interface with EUD or NTCP, they are not widely used in the daily practice because of the intangible choice of the biological parameters.

Table 1 shows that, with regard to most normal tissue factors, the plan with EUD was significantly better than the one without EUD, especially in decreasing the bladder doses as well as the related predicted toxicities. However, the negative impact on the tumor target coverage should not be ignored. Indeed, Table 1 shows that the $D_{\text {min }}, V_{76 \text { Gy }}$ and $\mathrm{HI}$ of $\mathrm{PTV}$ prostate are significantly decreased in $\operatorname{Plan}_{\text {bio }}$. The explanation is related to the PTV and OAR overlapped area, generating a conflict between the objectives. However, these slight changes in tumor targets appear to be moderate by looking at the average DVH curve (Figure 1), and all the dosimetric parameters finally fully respected the GETUG constraints. Moreover, the TCP values from physical and biological optimization schemes were not significantly different. Thus, the difference in the tumor target dose coverage may not affect the treatment benefits. Wu et al and Senthilkumar et al ${ }^{4,39,40}$ also pointed out that EUD-optimized IMRT may impact negatively on the PTV DVH curve (lower prescription dose coverage percentage and lower homogeneity versus higher hot spots on tumor). Li et al ${ }^{41}$ compared a biological IMRT optimization strategy to a physical IMRT optimization strategy among three commercial TPSs (Monaco, Pinnacle and Eclipse) and argued that although biological IMRT better spared normal tissues, the same dose coverage on target could hardly be achieved, compared to physical IMRT. This situation can also be observed in Figure 3, where the higher isodoses were "pushed" out of the bla-in and congregated in a smaller target area. The biological constraints should therefore be used with the assistance of physical constraints, especially for sparing serial organs (confining maximum dose on normal tissues). 
Table 1 also shows that the femoral head dose would be very slightly increased due to the beam angles, but remained very low $\left(V_{55} \%<0.4 \%\right)$ without having any clinical impact. VMAT or helical tomotherapy technique may play a positive role in avoiding the femora. Considering the recent new radiotherapy equipment, such as tomotherapy and cyberknife, treatment beams are much thinner (due to the small subfields or the thin circular collimators) and have higher degrees of freedom. ${ }^{42,43}$ If the separated bladder sub-volume EUD constraints really bring the benefit of sparing the internal bladder wall, tomotherapy and cyberknife will provide more specific OAR protection by taking advantage of the accurate beam ray, especially for avoiding high-dose injury during hypofractionation or radiosurgery treatment. ${ }^{44}$

\section{Conclusion}

Bladder wall sub-volume EUD constraints may be applied in the biological IMRT optimization to reduce bladder toxicity in prostate IMRT. The use of both physical and biological constraints in the optimization process may improve the IMRT dose distribution.

\section{Abbreviations}

bla-in, internal bladder wall; bla-ex, external bladder wall; CI, conformal index; CT, computed topography; DVH, dosevolume histogram; EUD, equivalent uniform dose; gEUD, general equivalent uniform dose; GETUG, Groupe d'Etude des Tumeurs Uro-Génitales; HI, homogeneity index; IMRT, intensity-modulated radiotherapy; NTCP, normal tissue complication probability; OARs, organs at risk; Plan $_{\text {bio }}$, IMRT plan optimized by biological EUD constraints; Plan ${ }_{\text {phys }}$ IMRT plan optimized by physical constraints; PTV, planning target volume; PTV prostate $_{\text {PTV of prostate only; PTV }}$ prostate+SV $_{\text {PTV }}$ of prostate and seminal vesicle; SV, seminal vesicles; TCP, tumor control probability; VMAT, volumetric-modulated arc therapy; TPS, treatment planning system.

\section{Acknowledgments}

We are grateful to Dr Huang Wei (Department of Radiation Oncology, Shandong Cancer Hospital \& Institute, Jinan, People's Republic of China) and Ms Chen Jiuhong (Accuray Medical Equipment, Shanghai, People's Republic of China) for reviewing the manuscript. The study was supported by the National Natural Science Foundation of China (Grant numbers 81301298, 81530060 and 81671785), the Taishan Scholar Foundation (Grant number ts20120505) and China Postdoctoral Science Foundation.

The abstract of this paper was presented at the American Academy of Pain Medicine 57th Annual Meeting \&
Exhibition (July 12, 2015, Anaheim, CA, USA) with the title "Benefit of EUD in Prostate IMRT Planning to Reduce Bladder Toxicity" as a poster discussion presentation with interim findings. The poster's abstract was published in "Poster Abstracts" in Medical Physics Journal with the title "SU-E-P-42: benefit of EUD in Prostate IMRT Planning to Reduce Bladder Toxicity" (http://scitation.aip.org/content/ aapm/journal/medphys/42/6/10.1118/1.4923976).

\section{Author contributions}

JZ carried out the biological IMRT planning, participated in the raw data collection and drafted the manuscript. AS carried out the statistical analysis and participated in English editing. $\mathrm{PH}$ participated in the design of the study. CL carried out the physical IMRT planning. OA participated in the raw data collection and English editing. HS participated in the design of the study and the statistical analysis. JC carried out the patient cases collection and contours delineation. BL participated in the coordination and double-checked the contours delineation. RDC conceived the study, coordinated and helped to draft the manuscript. All authors contributed toward data analysis, drafting and critically revising the paper and agree to be accountable for all aspects of the work.

\section{Disclosure}

The authors report no conflicts of interest in this work.

\section{References}

1. Niemierko A. A generalized concept of equivalent uniform dose (EUD). Med Phys. 1999;26(6):1100.

2. Ebert MA. Viability of the EUD and TCP concepts as reliable dose indicators. Phys Med Biol. 2000;45(2):441-457.

3. Fleming C, Kelly C, Thirion P, Fitzpatrick K, Armstrong J. A method for the prediction of late organ-at-risk toxicity after radiotherapy of the prostate using equivalent uniform dose. Int J Radiat Oncol Biol Phys. 2011;80(2):608-613.

4. Wu Q, Mohan R, Niemierko A, Schmidt-Ullrich R. Optimization of intensity-modulated radiotherapy plans based on the equivalent uniform dose. Int J Radiat Oncol Biol Phys. 2002;52(1):224-235.

5. Zhu J, Zhang ZC, Li BS, et al. Analysis of acute radiation-induced esophagitis in non-small-cell lung cancer patients using the Lyman NTCP model. Radiother Oncol. 2010;97(3):449-454.

6. Choi B, Deasy JO. The generalized equivalent uniform dose function as a basis for intensity-modulated treatment planning. Phys Med Biol. 2002;47(20):3579-3589.

7. Mattes MD, Lee JC, Elnaiem S, et al. A patient-specific approach to prostate cancer contouring through assessment of the overlap volume between PTV and OARs. Int J Radiat Oncol Biol Phys. 2013; 87(2):S699.

8. Zelefsky MJ, Fuks Z, Happersett L, et al. Clinical experience with intensity modulated radiation therapy (IMRT) in prostate cancer. Radiother Oncol. 2000;55(3):241-249.

9. Mott JH, Livsey JE, Logue JP. Development of a simultaneous boost IMRT class solution for a hypofractionated prostate cancer protocol. Br J Radiol. 2004;77(917):377-386.

10. Fiorino C, Valdagni R, Rancati T, Sanguineti G. Dose-volume effects for normal tissues in external radiotherapy: pelvis. Radiother Oncol. 2009;93(2):153-167. 
11. Dees-Ribbers HM, Betgen A, Pos FJ, Witteveen T, Remeijer P, van Herk M. Inter-and intra-fractional bladder motion during radiotherapy for bladder cancer: a comparison of full and empty bladders. Radiother Oncol. 2014;113(2):254-259.

12. Zelefsky MJ, Levin EJ, Hunt M, et al. Incidence of late rectal and urinary toxicities after three-dimensional conformal radiotherapy and intensitymodulated radiotherapy for localized prostate cancer. Int J Radiat Oncol Biol Phys. 2008;70(4):1124-1129.

13. Kupelian PA, Willoughby TR, Reddy CA, Klein EA, Mahadevan A. Hypofractionated intensity-modulated radiotherapy ( 70 Gy at 2.5 Gy per fraction) for localized prostate cancer: Cleveland Clinic experience. Int J Radiat Oncol Biol Phys. 2007;68(5):1424-1430.

14. Karlsdottir A, Muren LP, Wentzel-Larsen T, Dahl O. Late gastrointestinal morbidity after three-dimensional conformal radiation therapy for prostate cancer fades with time in contrast to genitourinary morbidity. Int J Radiat Oncol Biol Phys. 2008;70(5):1478-1486.

15. Cahlon O, Zelefsky MJ, Shippy A, et al. Ultra-high dose (86.4 Gy) IMRT for localized prostate cancer: toxicity and biochemical outcomes. Int J Radiat Oncol Biol Phys. 2008;71(2):330-337.

16. Lips IM, Dehnad H, van Gils CH, Boeken Kruger AE, van der Heide UA, van Vulpen M. High-dose intensity-modulated radiotherapy for prostate cancer using daily fiducial marker-based position verification: acute and late toxicity in 331 patients. Radiat Oncol. 2008;3:15.

17. Al-Mamgani A, Heemsbergen WD, Peeters ST, Lebesque JV. Role of intensity-modulated radiotherapy in reducing toxicity in dose escalation for localized prostate cancer. Int J Radiat Oncol Biol Phys. 2009;73(3):685-691.

18. Sanda MG, Dunn RL, Michalski J, et al. Quality of life and satisfaction with outcome among prostate-cancer survivors. $N$ Engl J Med. 2008;358(12):1250-1261

19. Zhu J, Simon A, Ospina JD, et al. 7007 POSTER DISCUSSION predictive models of bladder toxicity in prostate cancer radiotherapy. Eur J Cancer. 2011;47:S486-S486.

20. Thor M, Olsson C, Oh JH, et al. Urinary bladder dose-response relationships for patient-reported genitourinary morbidity domains following prostate cancer radiotherapy. Radiother Oncol. 2016;119(1):117-122.

21. Harsolia A, Vargas C, Yan D, et al. Predictors for chronic urinary toxicity after the treatment of prostate cancer with adaptive three-dimensional conformal radiotherapy: dose-volume analysis of a phase II dose-escalation study. Int J Radiat Oncol Biol Phys. 2007;69(4):1100-1109.

22. Baradaran-Ghahfarokhi M. Normal tissue complication probability modeling of radiation-induced bladder complications. J Radiobiol. 2014;1(1):19-20.

23. Cheung MR, Tucker SL, Dong L, et al. Investigation of bladder dose and volume factors influencing late urinary toxicity after external beam radiotherapy for prostate cancer. Int J Radiat Oncol Biol Phys. 2007;67(4):1059-1065.

24. Marks LB, Yorke ED, Jackson A, et al. Use of normal tissue complication probability models in the clinic. Int J Radiat Oncol Biol Phys. 2010;76(Suppl 3):S10-S19.

25. Bey P, Carrie C, Beckendorf V, et al. Dose escalation with 3D-CRT in prostate cancer: French study of dose escalation with conformal 3D radiotherapy in prostate cancer - preliminary results. Int J Radiat Oncol Biol Phys. 2000;48(2):513-517.

26. Beckendorf V, Guerif S, Le Prise E, et al. 70 Gy versus 80 Gy in localized prostate cancer: 5-year results of GETUG 06 randomized trial. Int J Radiat Oncol Biol Phys. 2011;80(4):1056-1063.

OncoTargets and Therapy

\section{Publish your work in this journal}

OncoTargets and Therapy is an international, peer-reviewed, open access journal focusing on the pathological basis of all cancers, potential targets for therapy and treatment protocols employed to improve the management of cancer patients. The journal also focuses on the impact of management programs and new therapeutic agents and protocols on
27. Burman C, Kutcher GJ, Emami B, Goitein M. Fitting of normal tissue tolerance data to an analytic function. Int J Radiat Oncol Biol Phys. 1991;21(1):123-135.

28. Dirscherl T, Alvarez-Moret J, Bogner L. Advantage of biological over physical optimization in prostate cancer? Z Med Phys. 2011;21(3): 228-235.

29. Valdagni R, Rancati T, Fiorino C. Predictive models of toxicity with external radiotherapy for prostate cancer: clinical issues. Cancer. 2009; 115(13):3141-3149.

30. Zhu J, Li BS, Shu HZ, et al. A method of exporting and reconstruction of dose volume histogram from Pinnacle TPS. Clin J Radiat Oncol. 2014;23(1):34-35.

31. Grégoire V, Mackie TR. State of the art on dose prescription, reporting and recording in intensity-modulated radiation therapy (ICRU report No. 83)[J]. Cancer/Radiothérapie. 2011;15(6):555-559.

32. Ospina JD, Zhu J, Chira C, et al. Random forests to predict rectal toxicity following prostate cancer radiation therapy. Int J Radiat Oncol Biol Phys. 2014;89(5):1024-1031.

33. Fowler JF. The linear-quadratic formula and progress in fractionated radiotherapy. Br J Radiol. 1989;62(740):679-694.

34. Wang JZ, Li XA, Yu CX, DiBiase SJ. The low alpha/beta ratio for prostate cancer: what does the clinical outcome of HDR brachytherapy tell us? Int J Radiat Oncol Biol Phys. 2003;57(4):1101-1108.

35. Fowler J, Chappell R, Ritter M. Is alpha/beta for prostate tumors really low? Int J Radiat Oncol Biol Phys. 2001;50(4):1021-1031.

36. Miften MM, Das SK, Su M, Marks LB. A dose-volume-based tool for evaluating and ranking IMRT treatment plans. J Appl Clin Med Phys. 2004;5(4):1-14.

37. Yoon M, Park SY, Shin D, et al. A new homogeneity index based on statistical analysis of the dose-volume histogram. J Appl Clin Med Phys. 2007;8(2):9-17.

38. Marks LB, Carroll PR, Dugan TC, Anscher MS. The response of the urinary bladder, urethra, and ureter to radiation and chemotherapy. Int J Radiat Oncol Biol Phys. 1995;31(5):1257-1280.

39. Wu Q, Djajaputra D, Wu Y, Zhou J, Liu HH, Mohan R. Intensitymodulated radiotherapy optimization with gEUD-guided dose-volume objectives. Phys Med Biol. 2003;48(3):279-291.

40. Senthilkumar K, Maria Das KJ, Balasubramanian K, Deka AC, Patil BR. Estimation of the effects of normal tissue sparing using equivalent uniform dose-based optimization. J Med Phys. 2016;41(2):123-128.

41. Allen Li X, Alber M, Deasy JO, et al. The use and QA of biologically related models for treatment planning: short report of the TG-166 of the therapy physics committee of the AAPM. Med Phys. 2012;39(3):1386-1409.

42. Kong M, Hong SE, Chang SG. Hypofractionated helical tomotherapy ( 75 Gy at 2.5 Gy per fraction) for localized prostate cancer: long-term analysis of gastrointestinal and genitourinary toxicity. Onco Targets Ther. 2014;7:553-566.

43. Seisen T, Drouin SJ, Phe V, et al. Current role of image-guided robotic radiosurgery (Cyberknife (R)) for prostate cancer treatment. BJU Int. 2013;111(5):761-766.

44. Chen LN, Suy S, Uhm S, et al. Stereotactic body radiation therapy (SBRT) for clinically localized prostate cancer: the Georgetown University experience. Radiat Oncol. 2013;8:58.

patient perspectives such as quality of life, adherence and satisfaction The manuscript management system is completely online and includes a very quick and fair peer-review system, which is all easy to use. Visit http://www.dovepress.com/testimonials.php to read real quotes from published authors. 